\title{
A TESTICULAR MASS AS THE SOLE MANIFESTATION OF IgG4- RELATED DISEASE
}

Vinicius de Castro Thomazi1,", Maria Lucia Lemos Lopes ${ }^{1}$, Tatiana Freitas Tourinho ${ }^{1}$, Maria Odete Esteves Hilario', Eduardo Rosa de Oliveira ${ }^{1}$, Thiago Willers ${ }^{1}$, Rafael Coradin ${ }^{1}$, Gilberto Scanagatta ${ }^{1}$, Raissa Velasques de Figueiredo ${ }^{1}$, Bruno Trevisan ${ }^{1}$, Gabriela Sasso Padilha ${ }^{1}$, Luana Ribeiro Carlos ${ }^{1}$

1. Universidade Federal de Ciências da Saúde de Porto Alegre, Porto Alegre (RS), Brazil.

*Corresponding author: gilberto_scanagatta@hotmail.com

\section{BACKGROUND}

IgG4-related disease (IgG4-RD) is a systemic immune-mediated syndrome characterized by diffuse enlargement or tumor-like masses in multiple organs. Histology typically shows infiltration by lgG4+ plasma cells and lymphocytes, fibrosis, and modest tissue eosinophilia. The sites most commonly affected include the pancreas, bile ducts, salivary glands, kidneys, and the retroperitoneum. Treatment is still largely based upon observational data, and randomized trials have yet to be performed to compare treatment strategies. Tests are uncommon sites of involvement, with only a few cases reported in the literature. We report the case of a patient with exclusive testicular involvement of IgG4-RD, manifesting as a growing solitary mass in the left testicle.

\section{CASE REPORT}

A 67-year-old male with a history of benign prostate enlargement presented with complaints of a nodular mass in the left testicle, with slowly progressive swelling of the scrotum. He denied fever or any other symptoms. Doppler ultrasonography of the scrotum showed an enlarged left testicle $\left(21.9 \mathrm{~cm}^{3}\right)$ with a heterogeneous echotexture and increased internal blood flow, suggestive of an inflammatory process or testicular lymphoma as the main differentials. Head, thorax and abdomen computed tomography (CT) scans showed no masses or signs of fibrosis in any other organ, but revealed signs of pulmonary embolism. Rivaroxaban was started for treatment of pulmonary embolism. Radical left orchiectomy was performed. Histology of the surgical specimen showed inflammation and nodular fibrosis, with plasma cells, histiocytes and eosinophils occupying nearly the entirety of the testicular parenchyma. Immunohistochemistry suggested IgG4-related disease. Cultures of the specimen were negative for fungi and mycobacteria. Prednisone $80 \mathrm{mg} /$ day PO was started, followed by gradual taper over 2 months until $60 \mathrm{mg} /$ day. The patient is currently followed by the rheumatology team.

\section{CONCLUSION}

IgG4-related disease is frequently a diagnostic and treatment challenge. Diagnosis is based on tissue biopsy accompanied by clinical, serologic, and/or radiologic data. Even though solitary involvement of the testes is uncommon, a growing number of "atypical" presentations are to be expected as clinical suspicion grows. The goal of therapy is to induce disease remission, but the best strategy for doing so has yet to be established. The customary initial therapy is prednisone, tapered to discontinuation based on clinical response and signs of remission. Relapses are common without treatment, and the alternative for glucocorticoid failure or contraindication is usually rituximab. 